\title{
Prognostic value of serum $\gamma$-glutamyl transferase in unresectable hepatocellular carcinoma patients treated with transcatheter arterial chemoembolization combined with conformal radiotherapy
}

\author{
DONG CHEN ${ }^{1,2}$, RENBEN WANG $^{1 *}$, XIANGJIAO MENG $^{1}$, HONGJIANG YAN $^{1}$, SHUMEI JIANG ${ }^{1}$,

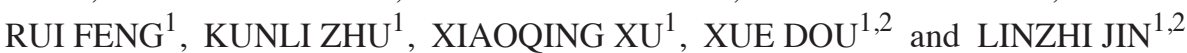 \\ ${ }^{1}$ Department of Radiation Oncology, Shandong Cancer Hospital; ${ }^{2}$ School of Medicine and Life Sciences, \\ University of Jinan-Shandong Academy of Medical Sciences, Shandong Cancer Hospital, Jinan, Shandong 250117, P.R. China
}

Received April 13, 2014; Accepted July 23, 2014

DOI: $10.3892 / 01.2014 .2456$

\begin{abstract}
The detection of $\gamma$-glutamyl transferase (GGT) has previously been reported to be useful in the diagnosis in hepatocellular carcinoma (HCC). The aim of the present study was to investigate the baseline serum GGT levels in patients with intermediate HCC (Barcelona Clinic Liver Cancer stage B) following treatment with transcatheter arterial chemoembolization (TACE) combined with three-dimensional conformal radiotherapy (3DCRT). A total of 154 intermediate HCC patients with Child-Pugh grade A were retrospectively investigated. Receiver operating characteristic (ROC) analysis was used to determine the optimal threshold for the GGT serum levels, and univariate and multivariate analyses were used to establish the prognostic factors. The median overall survival (OS) time was 24.3 months. The optimal threshold for GGT was $85 \mathrm{U} / \mathrm{L}$ (sensitivity, $75.13 \%$; specificity, $69.81 \%$; and area under the ROC curve, 0.763 ). The one-, three- and five-year OS rates were $79.9,49.7$ and $17.2 \%$, respectively, for patients with low GGT levels ( $\leq 85 \mathrm{U} / \mathrm{l})$ and 52.3, 22.1 and $8.5 \%$, respectively, for patients with high GGT levels ( $>85 \mathrm{U} / \mathrm{l})(\mathrm{P}=0.007)$. The results indicated that the serum GGT level was an independent prognostic factor (hazard ratio $=2.32 ; \mathrm{P}=0.007$ ) for OS. Furthermore, in subgroups stratified according to serum $\alpha$-fetoprotein, gross tumor volume and radiation dose, serum GGT was also found to correlate with OS $(\mathrm{P}<0.05)$. Therefore, the baseline GGT level may be a significant prognostic factor
\end{abstract}

Correspondence to: Professor Renben Wang, Department of Radiation Oncology, Shandong Cancer Hospital, 440 Jiyan Road, Jinan, Shandong 250117, P.R. China

E-mail:wangrenben@sina.cn

Key words: prognostic value, serum $\gamma$-glutamyl transferase, hepatocellular carcinoma, transcatheter arterial chemoembolization, embolization, three-dimensional conformal radiotherapy for intermediate HCC patients with Child-Pugh grade A following TACE combined with 3DCRT.

\section{Introduction}

Worldwide, hepatocellular carcinoma (HCC) is the fifth most prevalent type of cancer and, after lung and stomach cancer, is the third most common cause of cancer-related mortalities (1). Resection and liver transplantation are generally regarded as curative treatments for early-stage HCC and have exhibited effective results $(2,3)$. However, the majority of patients diagnosed with intermediate- to advanced-stage HCC receive only palliative treatment, such as transcatheter arterial chemoembolization (TACE). As the technology has developed, three-dimensional conformal radiotherapy (3DCRT) has allowed for high-dose radiation to be delivered to the target volume accurately, while minimizing the dose to normal liver tissues. TACE, combined with conventional external radiotherapy, has become the main treatment option for intermediate- to advanced-stage HCC, and the associated studies have reported safe and effective outcomes $(4,5)$.

$\gamma$-glutamyl transferase (GGT) is a cell surface heterodimeric glycoprotein, which is routinely tested for in clinical examinations. It is a simple biological marker which can be easily obtained from the patient at a low cost. High expression is observed in the biliary epithelium, brain capillaries and kidney tubules (6). According to Griffith et al (7), serum GGT can be used as a diagnostic biomarker for hepatobiliary disease, and GGT has been confirmed to be a major prognostic factor for survival in cirrhosis (8). Studies have demonstrated that serum GGT can predict tumor response and survival after TACE and surgery $(9,10)$; however, little is known regarding the prognostic role of GGT in treatment with combined TACE and 3DCRT. In the current study, 154 intermediate [Barcelona Clinic Liver Cancer (BCLC) stage B] (11) HCC patients were retrospectively investigated and the predictive value of the baseline serum GGT level with regard to overall survival (OS) was analyzed following the combined treatment. 


\section{Patients and methods}

Study design. The current retrospective study was conducted at the Department of Radiation Oncology at Shandong Cancer Hospital (Jinan, China). The criteria for entry into this study were as follows: i) HCC confirmed by liver biopsy or with the clinical features defined by the American Association for the Study of Liver Diseases [persistently elevated $\alpha$-fetoprotein (AFP) levels (>400 ng/ml) in conjunction with characteristic abdominal computed tomography (CT) or magnetic resonance imaging (MRI) with arterial phase enhancement and venous phase washout] (12); ii) all patients of intermediate stage (BCLC stage B) with Child-Pugh grade A according to the BCLC staging system (11); iii) Eastern Cooperative Oncology Group performance status of 0-1 (13); and iv) available follow-up data. The study protocol was approved by Shandong Tumor Prevention and Control Institutional Ethics Committee, Shandong Cancer Hospital and all patients provided written informed consent.

The clinical features of all patients included age, gender, tumor size, gross tumor volume (GTV), hepatitis virus infection, radiotherapy dose and total number of TACE treatments. The blood samples were obtained the morning prior to the TACE. Indicators of liver damage, including alanine transferase (ALT), GGT, albumin (ALB) and AFP, were systematically analyzed. The baseline imaging results (CT or MRI) of the liver were assessed within a week prior to TACE. For continuous variables, including age, GGT, tumor size, GTV and total number of TACE treatments, patients were divided into two groups according to the median values.

TACE procedures. TACE was performed using the conventional Seldinger technique (14). Hepatic and superior mesenteric artery angiographies were performed to identify the tumor vessel anatomy, tumor staining and the tumor-feeding artery. The catheter was superselectively inserted into the tumor-feeding artery in as close proximity as possible to the tumor. Chemotherapeutic agents, including $1.0 \mathrm{~g}$ 5-fluorouracil and $80 \mathrm{mg}$ cisplatin were infused, following which, an emulsion of $10 \mathrm{mg}$ mitomycin $\mathrm{C}$ and 5-30 ml lipiodol was administered. The dosage of chemotherapeutic agents or lipiodol was selected based on the tumor size, liver function and routine blood analysis. For large tumors that were hypervasculature in nature, a gelatin sponge was used for the further embolization of the tumor-feeding artery. TACE was performed every 1.5-2.0 months if required, on the basis of the tumor response and patient health.

3DCRT procedure. 3DCRT was performed two to four weeks after the final TACE course. A CT scan was initially performed for treatment planning. The patient position was fixed using vacuum casts in a supine position, with the arms raised above the head. GTV was delineated according to the primary lesion or lipiodol deposit from TACE. The clinical target volume was expanded by $5 \mathrm{~mm}$ on the basis of GTV and the planning target volume (PTV) was defined as GTV plus a 5-mm radial expansion, as well as a 10-mm craniocaudal expansion to account for daily setup error and respiratory organ motion (15). Organs at risk were also delineated, including the whole liver, non-target liver (whole liver minus PTV), stomach, kidney and spinal cord.
Aided by the beam's eye view, four to six coplanaror non-coplanar fields were designed. A cumulative dose-volume histogram was used to evaluate each treatment plan, and the target delineation was conducted by the same experienced oncologist. The median radiation dose was $45 \mathrm{~Gy}$ (range, 10-60 Gy) and the mean dose to normal liver was limited to $\leq 30 \mathrm{~Gy}$.

Evaluation of GGT and follow-up. The serum concentrations of GGT were analyzed using a Hitachi 917 machine (Roche Diagnostics, Mannheim, Germany). Tumor responses were evaluated with contrast-enhanced $\mathrm{CT}$ or MRI one month following TACE or 3DCRT. For patients without a complete response (CR), TACE was repeated. If the patients achieved a $\mathrm{CR}$, contrast-enhanced ultrasound, AFP test, CT and MRI were performed within three months following the treatment, and then routinely performed every six months until December 2013. In addition, routine blood analysis was conducted and liver function and serum tumor markers were also analyzed.

Statistical analysis. The software used for statistical analysis was SPSS 13.0 for Windows (SPSS Inc., Chicago, IL, USA). All consecutive results were presented as the mean \pm standard deviation. Comparison of variables was performed by the Mann-Whitney U test, $\chi^{2}$ test or Fisher's exact test. Variables that achieved statistical significance in the univariate analysis were subsequently included in a multivariate analysis using a stepwise forward Cox regression procedure to identify factors independently associated with mortality. OS was calculated as the interval between the time of the initiation of treatment and the time of mortality. The optimal threshold for GGT was identified by the receiver operating characteristic (ROC) curve, derived from a univariate logistic regression model predicting patient mortality prior to the median OS. This threshold served in all further uni- and multivariate analyses. Cumulative survival curves for each variable were obtained by using the Kaplan-Meier method and the difference was compared using the log-rank test. $\mathrm{P}<0.05$ was considered to indicate a statistically significant difference.

\section{Results}

Prognostic factors affecting survival. A total of 154 patients with intermediate HCC ( 71 females and 83 males) between January 2004 and December 2010 were included in the study. The median age and GTV were 55 years (range, 23-71 years) and $200 \mathrm{~cm}^{3}$. At the time of the analysis, the median number of TACE procedures performed for all patients was four (range, one to 10). According to the ROC analysis (Fig. 1), the optimal threshold for GGT was $85 \mathrm{U} / 1$. This resulted in a sensitivity of $75.13 \%$ and a specificity of $69.81 \%$ [area under the ROC curve, 0.763; 95\% confidence interval (CI), 0.645-0.880]. Furthermore, 115 patients $(74.7 \%)$ were included in the high GGT group, according to the cut-off level, and 39 patients $(25.3 \%)$ were included in the low GGT group.

The baseline characteristics of the 154 patients are summarized in Table I. The results indicated that GGT levels $(\mathrm{P}=0.003)$, ALT levels $(\mathrm{P}=0.012)$, ALB levels $(\mathrm{P}=0.038)$, GTV $(\mathrm{P}=0.002)$, AFP levels $(\mathrm{P}=0.01)$, total number of TACE procedures $(\mathrm{P}=0.039)$ and radiation dose $(\mathrm{P}=0.044)$ were all associated with OS. Factors exhibiting a significant difference 
Table I. Univariate analysis of factors associated with overall survival.

\begin{tabular}{|c|c|c|c|c|c|}
\hline \multirow[b]{2}{*}{ Risk factors } & \multirow[b]{2}{*}{$\mathrm{n}$} & \multicolumn{3}{|c|}{ Overall survival rate, $\%$} & \multirow[b]{2}{*}{ P-value } \\
\hline & & 1-year & 3-year & 5-year & \\
\hline Age, years & & & & & 0.772 \\
\hline$\leq 55$ & 60 & 70.0 & 31.7 & 13.3 & \\
\hline$>55$ & 94 & 58.5 & 21.3 & 9.6 & \\
\hline Gender & & & & & 0.144 \\
\hline Male & 83 & 71.1 & 42.2 & 14.5 & \\
\hline Female & 71 & 46.5 & 12.7 & 5.6 & \\
\hline Total bilirubin, $\mu \mathrm{mol} / 1$ & & & & & 0.586 \\
\hline$\leq 17.1$ & 60 & 61.7 & 31.7 & 8.3 & \\
\hline$>17.1$ & 94 & 43.6 & 19.1 & 7.4 & \\
\hline GGT, U/1 & & & & & 0.003 \\
\hline$\leq 85$ & 39 & 79.5 & 48.7 & 17.9 & \\
\hline$>85$ & 115 & 52.2 & 21.7 & 8.7 & \\
\hline Prothrombin time, sec & & & & & 0.388 \\
\hline$\leq 14$ & 121 & 66.9 & 38.8 & 9.1 & \\
\hline$>14$ & 33 & 33.3 & 15.2 & 3.0 & \\
\hline ALT, U/l & & & & & 0.012 \\
\hline$\leq 40$ & 74 & 63.5 & 31.1 & 9.5 & \\
\hline$>40$ & 80 & 45.0 & 20.0 & 8.8 & \\
\hline ALB, $g / 1$ & & & & & 0.038 \\
\hline$\leq 35$ & 43 & 32.6 & 18.6 & 4.7 & \\
\hline$>35$ & 111 & 57.7 & 33.3 & 7.2 & \\
\hline AFP, ng/ml & & & & & 0.010 \\
\hline $\mathrm{A} \leq 400$ & 92 & 60.9 & 51.1 & 14.1 & \\
\hline $\mathrm{B}>400$ & 62 & 40.3 & 11.3 & 3.2 & \\
\hline GTV, $\mathrm{cm}^{3}$ & & & & & 0.002 \\
\hline$\leq 200$ & 73 & 72.6 & 60.3 & 24.7 & \\
\hline$>200$ & 81 & 61.7 & 27.2 & 14.8 & \\
\hline Radiation dose, Gy & & & & & 0.044 \\
\hline$\leq 45$ & 82 & 56.1 & 18.3 & 4.9 & \\
\hline$>45$ & 72 & 61.1 & 36.1 & 16.7 & \\
\hline TACE, $n$ & & & & & 0.039 \\
\hline $1-4$ & 70 & 61.4 & 11.4 & 8.6 & \\
\hline$>4$ & 84 & 64.3 & 30.9 & 12.9 & \\
\hline $\mathrm{HBV}$ & & & & & 0.054 \\
\hline Positive & 89 & 49.4 & 20.2 & 4.5 & \\
\hline Negative & 65 & 50.8 & 38.5 & 12.3 & \\
\hline
\end{tabular}

GGT, $\gamma$-glutamyl transferase; ALT, alanine transferase; ALB, albumin; AFP, $\alpha$-fetoprotein; GTV, gross tumor volume; TACE, transcatheter arterial chemoembolization; HBV, hepatitis B virus.

by univariate analysis were adopted when multivariate Cox proportional-hazards analysis was performed. The results demonstrated that GGT levels $[\mathrm{P}=0.001$; hazard ratio $(\mathrm{HR})$, 2.32; 95\% CI, 1.133-3.643], GTV ( $\mathrm{P}=0.007$; HR, 1.263; 95\% CI, 1.361-7.401), AFP levels $(\mathrm{P}=0.006$; HR, 1.84; $95 \% \mathrm{CI}$, 1.218-3.059) and radiation dose $(\mathrm{P}=0.035 ; \mathrm{HR}, 1.75 ; 95 \% \mathrm{CI}$, 1.157-2.998) were independent risk factors for OS (Table II). A comparison of the clinical results between patients with low and elevated GGT expression is summarized in Table III. The results indicated that patients with elevated GGT usually had higher serum ALT, AFP and total bilirubin levels, as well as lower ALB and shorter prothrombin time.

OS of patients with various GGT levels. In Table IV, the median OS time following TACE combined with 3DCRT was 24.3 months (95\% CI, 12.84-35.16), with one-, three- and 
Table II. Multivariate analysis of factors associated with overall survival.

\begin{tabular}{lccc}
\hline Risk factors & Hazard ratio & 95\% CI & P-value \\
\hline GGT $(\leq 85 \mathrm{vs}>85 \mathrm{U} / \mathrm{l})$ & 2.320 & $1.133-3.643$ & 0.001 \\
$\mathrm{ALT}(\leq 40 \mathrm{vs}>40 \mathrm{U} / \mathrm{l})$ & 1.263 & $0.599-2.092$ & 0.545 \\
$\mathrm{ALB}(\leq 35 \mathrm{vs}>35 \mathrm{~g} / \mathrm{l})$ & 0.721 & $0.509-1.021$ & 0.065 \\
GTV $\left(\leq 200 \mathrm{vs}>200 \mathrm{~cm}^{3}\right)$ & 1.263 & $1.361-7.401$ & 0.007 \\
TACE, $\mathrm{n}$ & 0.648 & $0.381-1.101$ & 0.109 \\
Radiation dose, Gy & 1.750 & $1.157-2.998$ & 0.035 \\
AFP $(\leq 400 \mathrm{vs}>400 \mathrm{ng} / \mathrm{ml})$ & 1.840 & $1.218-3.059$ & 0.006 \\
\hline
\end{tabular}

GGT, $\gamma$-glutamyl transferase; ALT, alanine transferase; ALB, albumin; GTV, gross tumor volume; TACE, transcatheter arterial chemoembolization; AFP, $\alpha$-fetoprotein; CI, confidence interval.

Table III. Comparison of clinicopathological factors between patients with low and high $\gamma$-glutamyl transferase levels.

\begin{tabular}{lccc}
\hline Risk factors & Low GGT $(\leq 85 \mathrm{U} / \mathrm{l})$ & High GGT $(>85$ U/l) & P-value \\
\hline Gender, $\mathrm{n}$ & & 60 & 0.173 \\
Male & 23 & 44 & \\
Female & 27 & $51.7 \pm 14.5$ & 0.087 \\
Age, years & $50.2 \pm 12.1$ & $57.3 \pm 38.5$ & \\
ALT, U/l & $39.7 \pm 18.9$ & 65 & 0.023 \\
HBV & & 39 & 0.088 \\
Positive & 24 & $21831.2 \pm 18723.5$ \\
Negative & 26 & $11.22 \pm 2.24$ \\
AFP, ng/ml & $3112.1 \pm 3840.3$ & $35.3 \pm 4.1$ \\
Prothrombin time, sec & $12.53 \pm 1.75$ & $18.7 \pm 8.7$ \\
ALB, g/l & $40.1 \pm 5.8$ & 0.020 \\
Total bilirubin, $\mu \mathrm{mol} / \mathrm{l}$ & $16.8 \pm 6.8$ & 0.238 \\
\hline
\end{tabular}

Data are presented as the mean \pm standard deviation. ALT, alanine transferase; HBV, hepatitis B virus; AFP, $\alpha$-fetoprotein; ALB, albumin; GGT, $\gamma$-glutamyl transferase.

five-year OS rates of 62.1, 27.5 and $10.9 \%$, respectively. Fig. 2 shows the cumulative overall survival curve for patients with low ( $\leq 85 \mathrm{U} / \mathrm{l})$ and high GGT levels (>85 U/1). For HCC patients with low GGT levels $(\mathrm{n}=39)$, the median OS time was 35.0 months (95\% CI, 29.9-40.1) with 1-, 3- and 5-year survival rates of 79.9, 49.7 and $17.2 \%$, respectively. For patients with high GGT levels $(n=115)$, the median OS time was 18.0 months (95\% CI 12.3-23.7) with 1-, 3-, and 5-year survival rates of $52.3,22.1$ and $8.5 \%$, respectively. The OS time of low GGT patients was significantly longer than that of the elevated GGT group (Fig. 2; $\mathrm{P}=0.007$ ).

Considering the effects of the high AFP levels, large GTV and high radiation dose on OS, these factors were stratified to further clarify the prognostic significance of GGT levels. The results demonstrated that serum GGT levels correlated with OS time in the subgroup of low $(\leq 400 \mathrm{ng} / \mathrm{ml})$ and high $(>400 \mathrm{ng} / \mathrm{ml})$ serum AFP levels $(\mathrm{P}=0.015$ and 0.029 , respectively; Fig $3 \mathrm{~A}$ and $\mathrm{B})$. When the results were stratified according to GTV, patients with low serum GGT levels had a longer OS time compared with that of the high GGT level group ( $\mathrm{P}=0.013$ and 0.012 , respectively; Fig $3 \mathrm{C}$ and $\mathrm{D})$. For the patients receiving a low radiation dose $(\leq 45 \mathrm{~Gy})$, those with high GGT levels exhibited a shorter OS time compared with that of the low GGT group ( $\mathrm{P}=0.03$; Fig. 3E). In the high radiation dose group, a significant difference was also observed in OS time between patients with low and high GGT levels $(\mathrm{P}=0.01$; Fig. 3F $)$.

\section{Discussion}

Measurement of GGT levels has been investigated and developed as a liver function test for several decades $(14,15)$. Hann et al (18) reported that serum GGT levels may predict $\mathrm{HCC}$ risk and mortality in hepatitis $\mathrm{B}$ virus (HBV) patients. Guiu et al (19) suggested that a serum GGT level of $\geq 165 \mathrm{U} / \mathrm{L}$ was associated with shorter time to treatment failure and OS time following TACE. Zhang et al (10) revealed that the predictive value was stable, and even higher, when a threshold 
Table IV. Different $\gamma$-glutamyl transferase levels associated with OS.

\begin{tabular}{lccrrr}
\hline & & \multicolumn{3}{c}{ OS rate, $\%$} \\
\cline { 4 - 5 } GGT & $\begin{array}{c}\text { Median } \\
\text { OS, months }\end{array}$ & 1-year & 3-year & 5-year & \\
\hline All patients & 24.3 & 62.1 & 27.5 & 10.9 & $12.8-35.2$ \\
$\leq 85$ U/1 & 35.0 & 79.9 & 49.7 & 17.2 & $29.9-40.1$ \\
$>85$ U/1 & 18.0 & 52.3 & 22.1 & 8.5 & $12.3-23.7$ \\
\hline
\end{tabular}

CI, confidence interval; OS, overall survival.

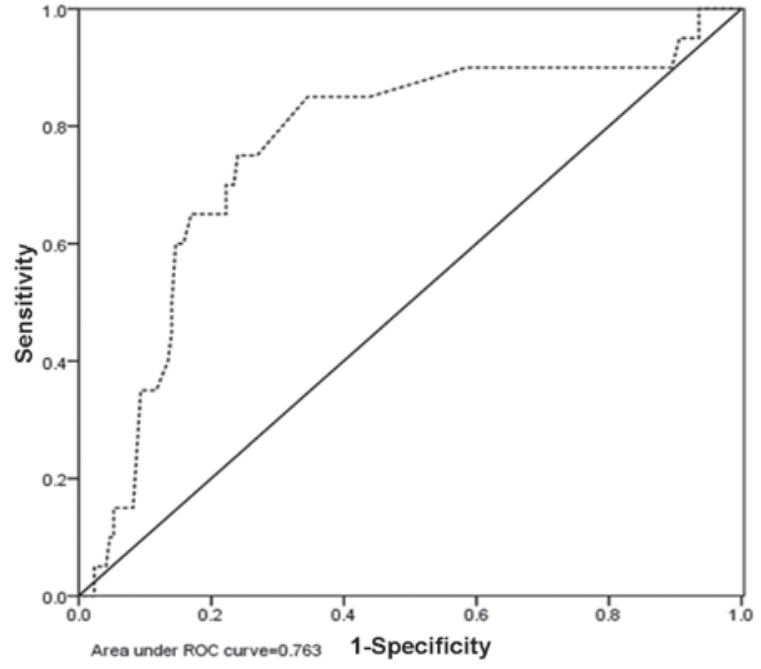

Figure 1. ROC curve derived from a univariate logistic regression model predicting patient mortality prior to the median overall survival time. The optimal threshold for $\gamma$-glutamyl transferase was $85 \mathrm{U} / 1$. This resulted in a sensitivity of $75.13 \%$ and a specificity of $69.81 \%$. Area under the ROC curve, $0.763 ; 95 \%$ confidence interval, $0.645-0.880$. ROC, receiver operating characteristic.

of between 60 and $300 \mathrm{U} / \mathrm{L}$ was used in a large retrospective study (277 patients). Furthermore, elevation of GGT levels was confirmed as a predictor of poor clinical outcome for intrahepatic cholangiocarcinoma patients (19). However, the correlation between GGT levels and TACE combined with 3DCRT remains unexplored. In the current study, the results demonstrated that GGT levels of $>85 \mathrm{U} / 1$ were associated with a shorter OS time $(\mathrm{P}=0.007)$. The optimal threshold of GGT levels (85 U/1) was identified by the ROC analysis (Fig. 1), derived from a univariate logistic regression model predicting patient mortality prior to the median OS.

The molecular mechanisms of GGT in HCC development remain unclear. It has been suggested that functions of the oxidative stress pathways in cellular response may mediate the role of GGT in tumorigenesis (21). The membrane-bound enzyme, GGT, catalyzes the degradation of extracellular glutathione (GSH), making the component amino acids available for the resynthesis of intracellular GSH (6). GSH can protect cells from damage induced by oxidants generated during normal metabolism. There is extensive evidence to suggest that GGT and GSH can cooperatively generate free radicals, subsequently leading to lipid peroxidation $(18,22,23)$. An additional explanation for the predictive

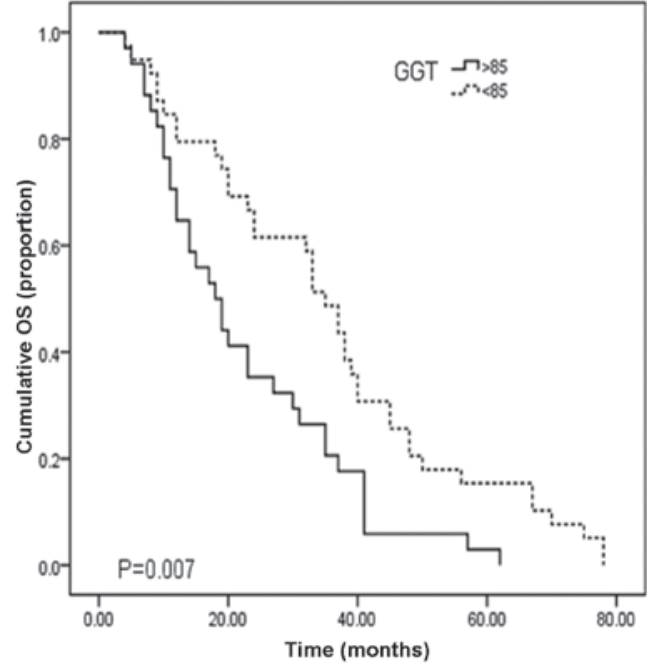

Figure 2. Cumulative OS curve of patients with low and high serum GGT levels (cut-off value was $85 \mathrm{U} / 1$ ). The one-, three- and five-year OS rates were 79.9, 49.7 and $17.2 \%$ for patients with low GGT levels $(\leq 85 \mathrm{U} / \mathrm{l})$, and $52.3,22.1$ and $8.5 \%$ for patients with high GGT levels (>85 U/L). OS, overall survival; GGT, $\gamma$-glutamyl transferase.

nature of GGT on OS of HCC patients in the current study is the significant implication of lipid peroxidation and other metabolisms in the tumorigenesis of a number of malignancies, including HCC $(24,25)$. Furthermore, an increased level of intracellular GSH often correlates with resistance to platinum-based drugs (26). Daubeuf et al (27) revealed that GGT activity may affect the cytotoxicity of platinum drugs in two ways: i) Following a reaction with the thiol group of cysteinylglycine, cisplatin can be detoxified extracellularly; or ii) in the case of carboplatin, GCT initiates the supply of GSH precursors, which subsequently increases the intracellular level of the tripeptide and provides enhanced defensive mechanisms to the cell. In the current study, cisplatin was the chemotherapeutic agent used during the TACE procedure. This may also explain the longer OS time of patients with low GGT levels $(\leq 85 \mathrm{U} / \mathrm{l})$ compared with those with high GGT levels (>85 U/1) as increased levels of intracellular GSH are often found to correlate with resistance to platinum-based drugs and a high level of GGT is associated with a higher concentration of GSH. Furthermore, in subgroups stratified according to serum AFP levels, GTV and radiation dose, GGT levels still had the power to discriminate patients with good results from those with poor outcomes. 

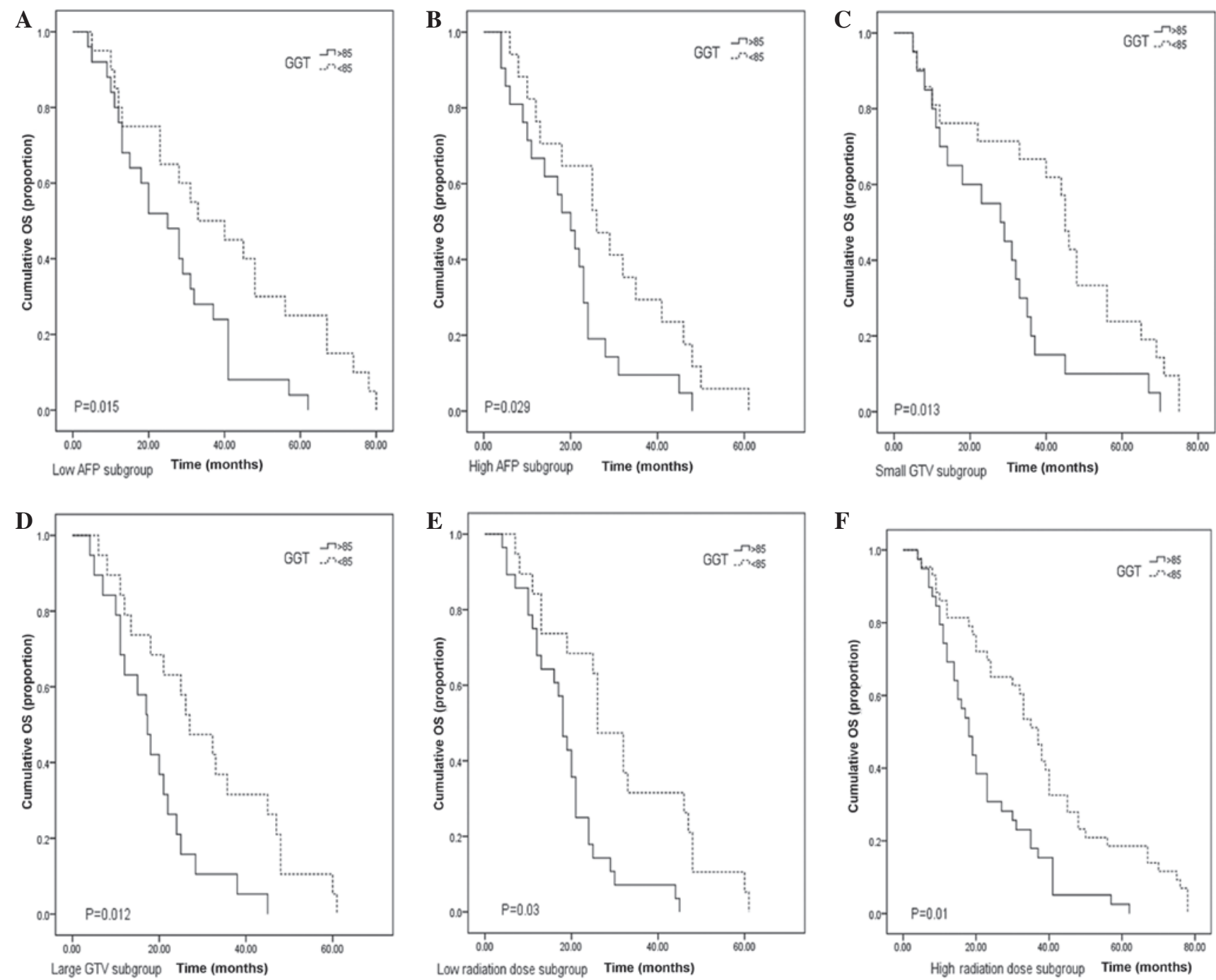

Figure 3. Kaplan-Meier estimates of OS rates for patients treated by transcatheter arterial chemoembolization with three-dimension conformal radiotherapy. The graphs represent the assessment of the prognostic role of GGT in subgroups, which were stratified according to (A and B) AFP levels ( $\leq 400 \mathrm{or}>400 \mathrm{ng} / \mathrm{ml}$, respectively), (C and D) GTV ( $\leq 200$ or $>200 \mathrm{~cm}^{3}$, respectively) and (E and F) radiation dose ( $\leq 45$ or $>45$ Gy, respectively). OS, overall survival; AFP, $\alpha$-fetoprotein; GTV, gross tumor volume; GGT, $\gamma$-glutamyl transferase.

In the present study, univariate analysis indicated that ALT levels, GTV, AFP levels, radiation dose and the number of TACE procedures all correlate with OS. In the multivariate analysis, only radiation dose, GTV and AFP levels were independent prognostic factors. The number of TACE procedures were not an independent predictive factor as different numbers of TACE were performed for each patient until the iodized oil deposited the whole tumor.

To date, radiotherapy technology has evolved markedly and is significant in the treatment of HCC. Kouloulias et al (28) reported that a high radiation dose (50-52 Gy) of 3DCRT can achieve a high local control rate in advanced HCC patients and inferior vena cava tumor thrombosis. In the current study, patients receiving a radiation dose of $>45$ Gy may achieve improved survival compared with those receiving a low radiation dose $(\mathrm{P}=0.035)$. According to Son et al (29) a large volume of liver receiving radiotherapy may lead to radiation-induced liver disease (RILD), which may result in hepatic failure and mortality. The authors suggested that in order to reduce the risk of RILD, the total liver volume receiving $<18$ Gy must be $>800 \mathrm{~cm}^{3}$; therefore, sparing more normal liver during radiotherapy is essential for HCC patients. In the current study, longer survival was observed in patients with smaller GTV $\left(\leq 200 \mathrm{~cm}^{3}\right)$ compared with that of larger GTV $(\mathrm{P}=0.013)$.

Cell proliferation and angiogenesis are promoted by AFP, as well as the increased resistance of cells toward tumor necrosis factor-associated, apoptosis-inducing ligand-induced apoptosis (30-32). It is well reported that AFP levels are a significant prognostic factor for patients following radiofrequency ablation and resection $(33,34)$. Tsai et al (35) and Kohles et al (36) demonstrated that AFP levels can be used as a biomarker to predict poor response following TACE. The current study indicated that serum AFP levels were an independent prognostic factor $(\mathrm{P}=0.006)$ for intermediate HCC patients treated with TACE combined with 3DCRT.

The present study had certain limitations, including the retrospective design and small number of patients. Therefore, 
further studies investigating larger patient populations are required to validate the results of the study.

In conclusion, the results presented in this study demonstrated that the baseline GGT levels of intermediate HCC patients with Child-Pugh grade A is an independent prognostic factor for OS following TACE combined with 3DCRT. In additon, the results of the present study may aid to predict outcomes for patients and may also be used to guide individualized treatment for HCC patients that receive TACE in combination with 3DCRT.

\section{References}

1. Cárdenes HR: Role of stereotactic body radiotherapy in the management of primary hepatocellular carcinoma. Rationale, technique and results. Clin Transl Oncol 11: 276-283, 2009.

2. Nathan H, Schulick RD, Choti MA and Pawlik TM: Predictors of survival after resection of early hepatocellular carcinoma. Ann Surg 249: 799-805, 2009

3. Cha CH, Saif MW, Yamane BH and Weber SM: Hepatocellular carcinoma: current management. Curr Probl Surg 47: 10-67, 2010

4. Zeng ZC, Tang ZY, Fan J, et al: A comparison of chemoembolization combination with and without radiotherapy for unresectable hepatocellular carcinoma. Cancer J 10: 307-316, 2004.

5. Xu LT, Zhou ZH, Lin JH, et al: Clinical study of transarterial chemoembolization combined with 3-dimensional conformal radiotherapy for hepatocellular carcinoma. Eur J Surg Oncol 37: 245-251, 2011.

6. Whitfield JB: Gamma glutamyl transferase. Crit Rev Clin Lab Sci 38: 263-355, 2001.

7. Griffith OW, Bridges RJ and Meister A: Transport of gamma-glutamyl amino acids: role of glutathione and gamma-glutamyl transpeptidase. Proc Natl Acad Sci USA 76: 6319-6322, 1979

8. Poynard T, Zourabichvili O, Hilpert G, et al: Prognostic value of total serum bilirubin/gamma-glutamyl transpeptidase ratio in cirrhotic patients. Hepatology 4: 324-327, 1984.

9. Ju MJ, Qiu SJ, Fan J, et al: Preoperative serum gamma-glutamyl transferase to alanine aminotransferase ratio is a convenient prognostic marker for Child-Pugh A hepatocellular carcinoma after operation. J Gastroenterol 44: 635-642, 2009

10. Zhang JB, Chen Y, Zhang B, et al: Prognostic significance of serum gamma-glutamyl transferase in patients with intermediate hepatocellular carcinoma treated with transcatheter arterial chemoembolization. Eur J Gastroenterol Hepatol 23 787-793, 2011

11. Llovet JM, Fuster J and Bruix J; Barcelona-Clínic Liver Cancer Group: The Barcelona approach: diagnosis, staging, and treatment of hepatocellular carcinoma. Liver Transpl 10: S115-S120, 2004

12. Bruix J, Sherman M, Llovet JM, et al; EASL Panel of Experts on HCC: Clinical management of hepatocellular carcinoma. Conclusions of the Barcelona-2000 EASL Conference. European Association for the Study of the Liver. J Hepatol 35: 421-430, 2001.

13. Oken MM, Creech RH, Tormey DC, et al: Toxicity and response criteria of the Eastern Cooperative Oncology Group. Am J Clin Oncol 5: 649-655, 1982

14. Saheb SM, Nath VN, Kumar KP and Padmaja PP: A novel method using Seldinger's technique for submental intubation in major craniomaxillofacial fractures: A case series. Indian J Anaesth 58: 48-50, 2014.

15. Kuo YC,Chiu YM, Shih WP, et al: Volumetricintensity-modulated Arc (RapidArc) therapy for primary hepatocellular carcinoma: comparison with intensity-modulated radiotherapy and 3-D conformal radiotherapy. Radiat Oncol 6: 76, 2011.

16. Whitfield JB, Pounder RE, Neale G and Moss DW: Serum-glytamyl transpeptidase activity in liver disease. Gut 13: 702-708, 1972

17. Idéo G, Morganti A and Dioguardi N: Gamma-glutamyl transpeptidase: a clinical and experimental study. Digestion 5: 326-336, 1972
18. Hann HW, Wan S, Myers RE, et al: Comprehensive analysis of common serum liver enzymes as prospective predictors of hepatocellular carcinoma in HBV patients. PloS one 7: e47687, 2012.

19. Guiu B, Deschamps F, Boulin M, et al: Serum gamma-glutamyl-transferase independently predicts outcome after transarterial chemoembolization of hepatocellular carcinoma: external validation. Cardiovasc Intervent Radiol 35: $1102-1108,2012$

20. Hanigan MH: gamma-Glutamyl transpeptidase, a glutathionase: its expression and function in carcinogenesis. Chem Biol Interact 111-112: 333-342, 1998.

21. Pompella A, Corti A, Paolicchi A, Giommarelli C and Zunino F: Gamma-glutamyltransferase, redox regulation and cancer drug resistance. Curr Opin Pharmacol 7: 360-366, 2007.

22. Stark AA, Zeiger E and Pagano DA: Glutathione metabolism by $\gamma$-glutamyl transpeptidase leads to lipid peroxidation: characterization of the system and relevance to hepatocarcinogenesis. Carcinogenesis 14: 183-189, 1993.

23. Paolicchi A, Tongiani R, Tonarelli P, Comporti $\mathrm{M}$ and Pompella A: gamma-Glutamyl transpeptidase-dependent lipid peroxidation in isolated hepatocytes and HepG2 hepatoma cells. Free Radic Biol Med 22: 853-860, 1997.

24. Negre-Salvayre A, Auge N, Ayala V, et al: Pathological aspects of lipid peroxidation. Free Radic Res 44: 1125-1171, 2010.

25. Zhao J, Zhao Y, Wang H, Gu X, Ji J and Gao C: Association between metabolic abnormalities and HBV related hepatocelluar carcinoma in Chinese: a cross-sectional study. Nutr J 10: 49, 2011.

26. Godwin AK, Meister A, O'Dwyer PJ, Huang CS, Hamilton TC and Anderson ME: High resistance to cisplatin in human ovarian cancer cell lines is associated with marked increase of glutathione synthesis. Proc Natl Acad Sci USA 89: 3070-3074, 1992.

27. Daubeuf S, Balin D, Leroy $\mathrm{P}$ and Visvikis A: Different mechanisms for gamma-glutamyltransferase-dependent resistance to carboplatin and cisplatin. Biochem Pharmacol 66: 595-604, 2003

28. Kouloulias V,MosaE,Georgakopoulos J,et al:Three-dimensional conformal radiotherapy for hepatocellular carcinoma in patients unfit for resection, ablation, or chemotherapy: A retrospective study. ScientificWorldJournal 2013: 780141, 2013.

29. Son SH, Choi BO, Ryu MR, et al: Stereotactic body radiotherapy for patients with unresectable primary hepatocellular carcinoma: dose-volumetric parameters predicting the hepatic complication. Int J Radiat Oncol Biol Phys 78: 1073-1080, 2010.

30. Li M, Zhou S, Liu X, Li P, McNutt MA and Li G: alpha-Fetoprotein shields hepatocellular carcinoma cells from apoptosis induced by tumor necrosis factor-related apoptosis-inducing ligand. Cancer Lett 249: 227-234, 2007.

31. Mitsuhashi N, Kobayashi S, Doki T, et al: Clinical significance of alpha-fetoprotein: involvement in proliferation, angiogenesis, and apoptosis of hepatocellular carcinoma. J Gastroenterol Hepatol 23: e189-e197, 2008

32. Yang X, Zhang Y, Zhang L, Zhang L and Mao J: Silencing alpha-fetoprotein expression induces growth arrest and apoptosis in human hepatocellular cancer cell. Cancer Lett 271: 281-293, 2008

33. Ho CM, Wu CY, Lee PH, Lai HS, Ho MC, Wu YM and Hu RH: Analysis of the risk factors of untransplantable recurrence after primary curative resection for patients with hepatocellular carcinoma. Ann Surg Oncol 20: 2526-2533, 2013.

34. Siripongsakun S, Wei SH, Lin S, et al: Evaluation of alpha-fetoprotein in detecting hepatocellular carcinoma recurrence after radiofrequency ablation. J Gastroenterol Hepatol 29: 157-164, 2014.

35. Tsai YJ, Hsu CY, Huang YH, et al: Early identification of poor responders to transarterial chemoembolization for hepatocellular carcinoma. Hepatol Int 5: 975-984, 2011.

36. Kohles N, Nagel D, Jüngst D, Durner J, Stieber P and Holdenrieder S: Prognostic relevance of oncological serum biomarkers in liver cancer patients undergoing transarterial chemoembolization therapy. Tumor Biol 33: 33-40, 2012. 